\title{
About an Ambiguity Appearing on the Hamiltonian Formulation of Quantum Mechanics
}

\author{
Gustavo V. López, Ana Griselda, Carlos R. Martínez-Prieto \\ Departamento de Física, Universidad de Guadalajara, Guadalajara, Mexico \\ Email: gulopez@cencar.udg.mx, an_248@hotmail.com, carlosrmtz57@gmail.com
}

How to cite this paper: López, G.V., Griselda, A. and Martínez-Prieto, C.R. (2018) About an Ambiguity Appearing on the Hamiltonian Formulation of Quantum Mechanics. Journal of Applied Mathematics and Physics, 6, 1382-1387. https://doi.org/10.4236/jamp.2018.66115

Received: April 25, 2018

Accepted: June 25, 2018

Published: June 28, 2018

Copyright ( 92018 by authors and Scientific Research Publishing Inc. This work is licensed under the Creative Commons Attribution International License (CC BY 4.0).

http://creativecommons.org/licenses/by/4.0/

\begin{abstract}
We study classical and quantum dynamics of the symmetric harmonic oscillator and the symmetric bouncer defined in 2-D. For these systems we get each of them for two different constants of motion, two Lagrangians and two Hamiltonians which describe the same classical dynamics. However, the quantization of these systems (using Schrödinger equation), using their two equivalents Hamiltonian, describes different quantum dynamics for each of them. This represents an ambiguity on the Hamiltonian formulation of the Quantum Mechanics.
\end{abstract}

\section{Keywords}

Harmonic Oscillator, Quantum Bouncer, Ambiguities in Quantum Mechanics Theory

\section{Introduction}

Modern Physics is based on Lagrangian or Hamiltonian [1] [2] [3] as mathematical objects to formulate and study the correspondent behavior of the natural systems. In particular, Quantum Mechanics has its foundations based on the Hamilton operator (generated from the Hamilton function of Classical Mechanics) and the Schrödinger's equation, which describes the linear evolution of the wave function. It is known that for most of the systems (conservative or including electromagnetic interaction) there is no problem to get a well unique Hamiltonian formulation [4] for the system. However, when one is dealing with dissipative systems, it has several problems [5] [6], and one of the main problems, which we are concerned, is that there can be two different Hamiltonian having the same classical behavior but different quantum behavior [7] [8]. In 
this paper, we want to point out that this type of ambiguity not only appears on dissipative systems, but it can also appear on conservative systems, and we focus on two conservative symmetric systems with two degrees of freedom: the harmonic oscillator and the bouncer. Firstly, we make the deduction of a pair of Hamiltonian for both systems, and this pair of Hamiltonians for each system describes the same classical dynamics. Then, we show that the associated quantum dynamics of each pair of Hamiltonian is different for each system.

\section{Classical Hamiltonians}

For the symmetric harmonic oscillator with two degrees of freedom, it is well known that a constant of motion and Hamiltonian can be given by

$$
K_{1}=\frac{m}{2}\left(v_{x}^{2}+v_{y}^{2}\right)+\frac{1}{2} m \omega^{2}\left(x^{2}+y^{2}\right)
$$

and

$$
H_{1}=\frac{p_{x}^{2}+p_{y}^{2}}{2 m}+\frac{1}{2} m \omega^{2}\left(x^{2}+y^{2}\right),
$$

where $m$ is the mass of the particle, and $\omega$ its angular frequency, being the same in both directions. However, it is not difficult to see that

$$
K_{2}=m v_{x} v_{y}+m \omega^{2} x y
$$

is also a constant of motion $\left(\mathrm{d} K_{2} / \mathrm{d} t=0\right)$ since it leads us to the equations of motion $m \dot{v}_{x}+m \omega^{2} x=0$ and $m \dot{v}_{y}+m \omega^{2} y=0$. Once we have this constant of motion, we use the known expression [9] [10] [11],

$$
L_{2}=v_{x} \int^{v_{x}} \frac{K_{2}\left(x, y, v_{x}, c v_{x}\right)}{v_{x}^{2}} \mathrm{~d} v_{x},
$$

to get the Lagrangian knowing the constant of motion. By substituting (3) in (4), one gets

$$
L_{2}=m v_{x} v_{y}-m \omega^{2} x y
$$

and the associated Hamiltonian $\left(H_{2}=v_{x} p_{x}+v_{y} p_{y}-L_{2}\right)$ is [12]

$$
H_{2}=\frac{p_{x} p_{y}}{m}+m \omega^{2} x y \text {. }
$$

In this way, we have obtained two different Hamiltonians which describe the same classical dynamics.

Consider now a symmetric bouncer with two degrees of freedom, characterized by the equations of motion

$$
m \ddot{x}=-f \text { and } m \ddot{y}=-f,
$$

where $f$ is a constant force, and $m$ is the mass of the particle. The well known constant of motion and Hamiltonian for this system are [13]

$$
\tilde{K}_{1}=\frac{m}{2}\left(v_{x}^{2}+v_{y}^{2}\right)+(x+y) f
$$

and 


$$
\tilde{H}_{1}=\frac{p_{x}^{2}+p_{y}^{2}}{2 m}+(x+y) f .
$$

Now, it is not difficult to see that

$$
\tilde{K}_{2}=m v_{x} v_{y}+(x+y) f
$$

is also a constant of motion of the system (7). Then, using (4), one gets the Lagrangian

$$
\tilde{L}_{2}=m v_{x} v_{y}-(x+y) f,
$$

and therefore, the new Hamiltonian of the system

$$
\tilde{H}_{2}=\frac{p_{x} p_{y}}{m}+(x+y) f .
$$

So, once again we have obtained two different Hamiltonian which describe the same classical dynamics.

\section{Quantization}

The symmetric 2-D harmonic oscillator and symmetric 2-D bouncer represent autonomous systems (Hamiltonians do not depend explicitly on time). So, solving the Schrödinger's equation [14],

$$
i \hbar \frac{\partial|\Psi\rangle}{\partial t}=\hat{H}|\Psi\rangle
$$

for the associated Hermitian Hamiltonian operator $\hat{H}$, is reduced through the transformation

$$
|\Psi\rangle=\mathrm{e}^{-i E t / \hbar}|\Phi\rangle,
$$

to an eigenvalue problem

$$
\hat{H}|\Phi\rangle=E|\Phi\rangle .
$$

Therefore, it is enough to know the eigenvalues of each pair of Hamiltonians to see whether or not the quantum dynamics described by these Hamiltonian are different.

\subsection{2-D Harmonic Oscillator}

The solution of the 2-D symmetric harmonic oscillator eigenvalue problem is well known, and it is given by

$$
E_{n_{1} n_{2}}^{(1)}=\hbar \omega(n+1), \quad\left\langle\mathbf{x} \mid n_{1} n_{2}\right\rangle=\prod_{i=1}^{2} c_{n_{i}} \mathrm{e}^{-\alpha x^{2} / 2} H_{n_{i}}\left(\alpha x_{i}\right),
$$

where $n$ is a non negative integer, $n=n_{1}+n_{2} \in \mathcal{Z}^{+}, \quad c_{n_{i}}$ is a constant, $c_{n_{i}}=\sqrt{\alpha / \sqrt{\pi} 2^{n_{i}} n_{i} !}$, and $\alpha$ is the constant defined as $\alpha=\sqrt{m \omega / \hbar}$. The full solution is

$$
|\Psi\rangle=\sum_{n_{1}, n_{2}=0} C_{n_{1} n_{2}} \mathrm{e}^{-i \omega(n+1) t}\left|n_{1} n_{2}\right\rangle \text {, with } \sum_{n_{1}, n_{2}=0}\left|C_{n_{1} n_{2}}\right|^{2}=1,
$$

where the coefficient $C_{n_{1} n_{2}}$ represents the amplitude of probability and can be 
determined by the initial conditions. The system is degenerated because of the symmetry, and the ground state is the state $|00\rangle$.

For the Hamiltonian (6), let us make the following change of variables

$$
\xi=\frac{1}{\sqrt{2}}(x+y) \text { and } \eta=\frac{1}{\sqrt{2}}(x-y) .
$$

So, the Hermitian Hamiltonian becomes

$$
\hat{H}_{2}=\frac{\hat{p}_{\xi}^{2}-\hat{p}_{\eta}^{2}}{2 m}+\frac{1}{2} m \omega^{2}\left(\xi^{2}-\eta^{2}\right),
$$

where the operators $\hat{p}_{\xi}$ and $\hat{p}_{\eta}$ are defined as $\hat{p}_{\xi}=-i \hbar \partial / \partial \xi$ and $\hat{p}_{\eta}=-i \hbar \partial / \partial \eta$. This Hamiltonian is just the difference of two 1-D symmetric harmonic oscillators, $\hat{H}_{2}=\hat{H}_{\xi}^{(h o)}-\hat{H}_{\eta}^{(h o)}$. This means that one has the following solution for the eigenvalue problem

$$
E_{n_{1} n_{2}}^{(2)}=\hbar \omega\left(n_{1}-n_{2}\right), \quad\left\langle\mathbf{x} \mid n_{1} n_{2}\right\rangle=\Phi_{n_{1}}\left(\frac{x+y}{\sqrt{2}}\right) \Phi_{n_{2}}\left(\frac{x-y}{\sqrt{2}}\right),
$$

where the function $\Phi_{n}(z)$ is defined as $\Phi_{n}(z)=c_{n} \mathrm{e}^{-\alpha x^{2} / 2} H_{n}(\alpha z)$, with the constants $c_{n}$ and $\alpha$ defined as before. Denoting the state defined by the product of the function of (20) as $\left|n_{1} n_{2}\right\rangle_{2}$, the full solution is written as

$$
|\Psi\rangle=\sum_{n_{1}, n_{2}=0} D_{n_{1} n_{2}} \mathrm{e}^{-i \omega\left(n_{1}-n_{2}\right) t}\left|n_{1} n_{2}\right\rangle_{2}, \quad \text { with } \sum_{n_{1}, n_{2}=0}\left|D_{n_{1} n_{2}}\right|^{2}=1 \text {. }
$$

The degeneration is infinity and there is not ground state of the system. Therefore, the quantum dynamics describe by (17) and (21) are completely different.

\subsection{2-D Bouncer}

For the usual Hamiltonian associated to the bouncer (9), one sees that this one can be written of the form

$$
\tilde{H}_{1}=\tilde{H}_{x}+\tilde{H}_{y}
$$

where $\tilde{H}_{x}$ and $\tilde{H}_{y}$ are defined as

$$
\tilde{H}_{x}=p_{x}^{2} / 2 m+f x \text { and } \tilde{H}_{y}=p_{y}^{2} / 2 m+f y,
$$

corresponding to the symmetric bouncer on each direction. The quantum bouncer eigenvalues are given in terms of the zeros of the Airy function [13]. Therefore, the solution of the eigenvalue problem $\tilde{H}_{1}|\Phi\rangle=E|\Phi\rangle$ is given by

$$
\tilde{E}_{n_{1} n_{2}}^{(1)}=f l_{f}\left(z_{n_{1}}+z_{n_{2}}\right), \quad A i\left(-z_{n_{i}}\right)=0, \quad l_{f}=\left(\frac{\hbar^{2}}{2 m f}\right)^{1 / 3} .
$$

The eigenfunctions are

$$
\tilde{\Phi}_{n_{1} n_{2}}=\left\langle\mathbf{x} \mid n_{1} n_{2}\right\rangle=\frac{A i\left(z_{1}-z_{n_{1}}\right)}{\left|A^{\prime} i\left(-z_{n_{1}}\right)\right|} \cdot \frac{A i\left(z_{2}-z_{n_{2}}\right)}{\left|A^{\prime} i\left(-z_{n_{2}}\right)\right|},
$$

where $z_{1}$ and $z_{2}$ are defined as $z_{1}=x / l_{f}$ and $z_{2}=y / l_{f}, A^{\prime} i$ represents 
the differentiation of the Airy function. The full Scrödinger solution is

$$
|\Psi\rangle=\sum_{n_{1}, n_{2}} \tilde{C}_{n_{1} n_{2}} \mathrm{e}^{-i f f_{f}\left(z_{n_{1}}+z_{n_{2}}\right) t / \hbar}\left|n_{1} n_{2}\right\rangle \text {, with } \sum_{n_{1} n_{2}}\left|C_{n_{1} n_{2}}\right|^{2}=1 .
$$

The spectrum is discrete, there is degeneration (because of the symmetry), and there is a ground state for the system.

Now, for the bouncer Hamiltonian (12), we make the same change of variables (18), bringing about the Hamiltonian operator

$$
\hat{H}_{2}=\frac{\hat{p}_{\xi}^{2}-\hat{p}_{\eta}^{2}}{2 m}+f \sqrt{2} \xi \text {. }
$$

This Hamiltonian represents a quantum bouncer in the variable $\xi$, and a free particle motion in the variable $\eta$. The eigenvalues of this Hamiltonian are

$$
\tilde{E}_{n k}^{(2)}=\sqrt{2} f l_{f}^{*} z_{n}+\frac{\hbar^{2} k^{2}}{2 m}, \quad \text { with } l_{f}^{*}=\left(\frac{\hbar^{2}}{2 \sqrt{2} m f}\right)^{1 / 3},
$$

where $z_{n}$ is the zero of the Airy function, and $k$ is a continuous real constant. Its eigenfunctions are

$$
\tilde{\Phi}_{n k}(\mathbf{x})=\langle\mathbf{x} \mid n k\rangle=\frac{A i\left(z-z_{n}\right)}{\left|A^{\prime} i\left(-z_{n}\right)\right|} \mathrm{e}^{i k(x-y) / \sqrt{2}},
$$

where the variable $z$ is $z=\xi / l_{f}^{*}=(x+y) / l_{f}^{*} \sqrt{2}$, and the full solution of the Schrödinger's equations is

$$
|\Psi\rangle=\sum_{n} \int \mathrm{d} k C_{n}(k) \mathrm{e}^{-i \tilde{E}_{n k}^{(2)}}|n k\rangle \text {, with } \sum_{n} \int \mathrm{d} k\left|C_{n}(k)\right|^{2}=1 .
$$

The spectrum has a discrete component and a continuous component, and there is a ground state of the system for $k=0$ and $n=1$. As one can see from (26) and (30) the quantum dynamics is totally different, and this result was unknown until now.

\section{Conclusion}

We have shown that for two conservative 2-D symmetrical systems (harmonic oscillator and bouncer), we can find for each of them at least two different Hamiltonians describing the same classical dynamics. However, their quantum dynamics associated to each equivalent Hamiltonian is totally different, representing an ambiguity in the Hamiltonian formulation of the Quantum Mechanics. It is our guess that this type of ambiguity is intrinsic of the Hamiltonian theory, and it could be present on any quantum system.

\section{References}

[1] Cohen-Tannoudji, C., Diu, B. and Lalo e, F. (1997) Quantum Mechanics, I, II. John Wiley \& Sons, New York.

[2] Huang, H. (1963) Statistical Mechanics. John Wiley, New York.

[3] Schweber, S.S. (2005) An Introduction to Relativistic Quantum Field Theory. Dover Publications Inc., New York. 
[4] Dirac, P.A.M. (1976) The Principles of Quantum Mechanics. Oxford University Press, Oxford.

[5] Glauber, R. and Man'ko, V.I. (1984) Damping and Fluctuations in Couple Quantum Oscillator Systems. Soviet Physics JETP, 60, 450.

[6] Lopez, G. (1998) Ambiguities Appearing in the Study of Time-Dependent Constants of Motion for the One-Dimensional Harmonic Oscillator. International Journal of Theoretical Physics, 37, 1617-1623. https://doi.org/10.1023/A:1026628221912

[7] Dodonov, V.P., Man'ko, V.I. and Skarzhinsky, V.D. (1981) Classically Equivalent Hamiltonians and Ambiguities of Quantization: A Particle in a Magnetic Field. Hadronic Journal, 4, 1734.

[8] Lopez, G., Lopez, P. and Lopez, X.E. (2011) Ambiguities on the Hamiltonian Formulation of the Free Falling Particle with Quadratic Dissipation. Advanced Studies in Theoretical Physics, 5, 253.

[9] Kobusen, J.A. (1979) Some Comments on the Lagrangian Formalism for Systems with General Velocity Dependent Forces. Acta Physica Austriaca, 51, 193.

[10] Leubner, C. (1987) Inequivalent Lagrangians from Constants of the Motion. Physical Review A, 86, 9.

[11] Lopez, G. (1996) One-Dimensional Autonomous Systems and Dissipative Systems. Annals of Physics, 251, 372. https://doi.org/10.1006/aphy.1996.0118

[12] Montesinos, M. and Torres del Castillo, G.F. (2004) Symplectic Quantization, Inequivalent Quantum Theories, and Heisenberg's Principle of Uncertainty. Physical Review A, 70, 032104-1. https://doi.org/10.1103/PhysRevA.70.032104

[13] Lopez, G. and Gonzalez, G. (2004) Quantum Bouncer with Dissipation. International Journal of Theoretical Physics, 43, 1999-2008. https://doi.org/10.1023/B:IJTP.0000049005.73750.c0

[14] Messiah, A. (1967) Quantum Mechanics, I, II. North-Holland Publishing Co., Amsterdam. 